\title{
Stress 'Takotsubo' cardiomyopathy: questions still remain
}

\author{
Masunori Matsuzaki
}

Stress or 'Takotsubo' cardiomyopathy is a novel heart syndrome characterized by a transient (reversible) left ventricular apical dysfunction. The syndrome was first described in Japan and named 'Takotsubo' cardiomyopathy because the shape of the left ventricular apical ballooning observed resembled a 'tako-tsubo', Japanese for octopus pot or trap. Takotsubo cardiomyopathy is frequently associated with electrocardiographic changes (deep inverted $\mathrm{T}$ waves in precordial chest leads, with QT interval prolongation) and chest pain, which can closely resemble acute myocardial infarction or evolving acute coronary syndrome. Major impairment in left ventricular dysfunction is characterized by a distinctive ballooning appearance of the mid and apical left ventricle, sparing in most cases only the hyperkinetic left ventricular basal region. Many studies investigating selective coronary angiography performed in individuals immediately after the onset of symptoms have found no definite evidence of epicardial coronary obstruction. The precise clinical features and the etiologic basis of Takotsubo cardiomyopathy remain unclear, and there is diagnostic difficulty in clinically differentiating this syndrome from acute myocardial infarction. Also, this syndrome can occur during the clinical course of various systemic diseases.

Recently, many investigations have reported emotional stress as the predisposing factor for Takotsubo cardiomyopathy and have demonstrated that endogenous catecholamine levels have a crucial role in the development of this syndrome. Aggravation of various systemic disorders, such as cerebrovascular disease and bronchial asthma, and emotional or physical problems, such as the death of a close relative or friend, confrontation, domestic abuse, or devastating losses in

The precise
clinical features
and the
etiologic basis
of Takotsubo
cardiomyopathy
remain unclear...

business are considered to be possible triggering risk factors for the syndrome. A recent report has shown that the number of patients with acute-onset Takotsubo cardiomyopathy markedly increased immediately following the large earthquake (registering 6.8 on the Richter scale) that occurred in Niigata Prefecture, Japan, in 2004. Interestingly, 11 (69\%) of the 16 patients diagnosed as having Takotsubo cardiomyopathy within 1 month of the earthquake developed symptoms on the day of the earthquake. This finding supports the hypothesis that exposure to emotional or severe external stress might be a trigger for this heart syndrome.

The neurogenic stunned myocardium seen in some individuals following an acute cerebrovascular accident and histological changes in catecholamine-induced cardiomyopathy observed during endocrine crisis in patients with pheochromocytoma show similarities to the myocardial damage seen in those with stress cardiomyopathy, probably because of the markedly enhanced sympathetic activities in all cases. Furthermore, transient myocardial ischemia caused by intramyocardial microvascular spasm is thought to be one of the possible etiological mechanisms of this syndrome.

Previous reports from Japan have described a striking predilection of this cardiomyopathy for women of advanced age, which is consistent with the approximately sixfold femalemale predominance. Prompt and aggressive pharmacological and hemodynamic support during the acute phase can achieve rapid reversal of left ventricular dysfunction and adverse electrocardiographic changes, and inhospital prognosis is generally good with appropriate therapy. Nevertheless, further research into this increasingly reported condition is clearly needed. 